\title{
Malignant Pleural Mesothelioma: From the Bench to the Bedside
}

\author{
P. Astoula, b E. Roca ${ }^{\text {a }}$ F. Galateau-Salle ${ }^{c, d} \quad$ A. Scherpereel ${ }^{e-g}$ \\ ${ }^{a}$ Department of Thoracic Oncology, Pleural Diseases and Interventional Pulmonology, Hôpital Nord, and \\ ${ }^{\mathrm{b}}$ Aix-Marseille Université, Marseille, 'Service d'Anatomie Pathologique and d Mesopath Group, ERI 3 INSERM,

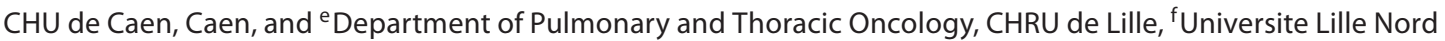 \\ de France, and 9INSERM U1019, Institut Pasteur de Lille, Lille, France
}

\section{Key Words}

Diagnosis - Mesothelioma - Pleura - Translational research • Treatment $\cdot$ Tumor markers $\cdot$ Targeted therapy

\begin{abstract}
Optimal management of malignant pleural mesothelioma (MPM), which is mainly based on patient characteristics and clinical stage, is not clearly defined yet, although detailed, practical guidelines for these patients have been proposed by some scientific societies. Translational research, in the field of this disease, is currently in progress and different molecular oncogenic pathways leading to the growth and progression of MPM have been characterized with recent pharmaceutical developments. However, further in-depth analysis still needs to be done for a more advanced deciphering of the step-by-step process leading from early increased mesothelial cell proliferation to invasive mesothelioma, from which we are expecting the development of definitively effective therapy. Thus, this review is an overview of the recent advances in the biology of MPM and their potential therapeutic applications in the field of MPM diagnosis and treatment.
\end{abstract}

\section{Introduction}

Malignant pleural mesothelioma (MPM) is a rare but highly aggressive tumor with a poor prognosis and increasing incidence. An optimal management of MPM is not clearly defined yet, although detailed, practical guidelines for these patients have been proposed by some scientific societies. These guidelines emphasize the numerous pitfalls in MPM diagnosis, and the need of innovative therapies and tools for monitoring MPM patients based on the limited and quite inconclusive results of current treatment.

Although the prospects for mesothelioma are rather pessimistic, recently research on MPM pathogenesis and biology exhibited great advances, leaving hope for significant advances in the management of these patients in the future.

Previous articles in this series: 1 . Anevlavis S, Tzouvelekis A, Bouros D: Mechanisms of pleural involvement in orphan diseases. Respiration 2012;83:5-12. 2. Rodriguez-Panadero F, Montes-Worboys A: Mechanisms of pleurodesis. Respiration 2012;83:91-98. 3. Grundy S, Bentley A, Tschopp J-M: Primary spontaneous pneumothorax: a diffuse disease of the pleura. Respiration 2012;83:185-189. 4. Haas AR, Sterman DH: Novel intrapleural therapies for malignant diseases. Respiration 2012;83:277-292. 5. Froudarakis ME: Pleural effusion in lung cancer: more questions than answers. Respiration 2012;83:367-376. 
Thus, this review is an overview of the recent progress in the biology of MPM and its potential therapeutic applications in cancer diagnosis and treatment. The chapter References was kept exhaustive to offer the reader the main studies in this field.

\section{Search Strategy}

A systematic analysis of the literature (2000-2012) was carried out using the databases Medline (National Library of Medicine, USA) with the following key words: mesothelioma and malignant pleural mesothelioma in combination with biology or targeted therapy. A manual search and review of reference lists in recent and relevant publications were also done in order to select the articles published in the literature. Therefore, abstracts from oncology meetings were not included in the reference list of this review.

\section{From the Bench: The Biology of MPM}

A better knowledge of the pathogenesis and molecular alterations in MPM represent a useful tool to develop predictive or prognostic biomarkers and potential therapeutic agents.

Asbestos fiber exposure is widely accepted as the main cause of MPM although exposure to other mineral fibers such as erionite, exposure to SV40 or radiation has been reported in low evidence-based reports. Although the mechanisms remain to be further elucidated, previous studies reported that the event is dependent on tumor necrosis factor (TNF)- $\alpha$ release by macrophages due to contact with an asbestos fiber. Subsequently, mesothelial cells express TNF- $\alpha$ receptor, and its interaction with ligand determines nuclear factor- $\kappa \mathrm{B}$ pathway activation and resistance of cancer cells to death $[1,2]$. Moreover, mesothelial cells are able to release reactive oxygen and nitrogen species (ROS and RNS) and high-mobilitygroup box one protein (HMGB1) leading to chronic inflammation, DNA damage and aneuploidy [3]. Asbestosinduced carcinogenesis is therefore based on a close relationship between chronic inflammatory processes and asbestos-induced mesothelial cell death offering potential targets for the prevention of mesothelioma by inhibition of the release of these molecules or the neutralization of their activity.

Other molecules, in particular cytokines [interleukin (IL)-6 and IL-8] and growth factors [vascular endothelial growth factor (VEGF), hepatocyte growth factor, transforming growth factor (TGF)- $\beta$, platelet-derived growth factor (PDGF) and insulin-like growth factor (IGF)], also implicated in MPM carcinogenesis may represent other targets for the management of MPM [see references 4-7 for details].

In patients with MPM, the following molecular alterations have been noted: chromosome alterations in tumor suppressor genes, allele loss $[8,9]$, gene silencing by DNA methylation in specific chromosomal regions [10], epigenetic deregulation of tumor suppressor genes by histone acetyltransferase and deacetylase (HDAC) chromatin condensation/decondensation balance [11--13] and gene mutations (leading to functional inactivation of NF2 for instance) usually found in case of loss of heterozygosity of 22q12 [14-19].

\section{Tumor Markers}

Numerous markers have been evaluated for the management of mesothelioma, but many discrepancies exist in the results obtained from different studies, as underlined in a recent review [20].

\section{Diagnostic Value}

Currently, there are a lot of markers based on the increasing understanding of the molecular and biological pathways of mesothelioma. Immunohistochemical markers have been tested for their potential to establish a diagnosis of mesothelioma on cytological grounds [21]. Genetic markers and serum markers are other promising tools, e.g. soluble mesothelin-related protein (SMRP) and megakaryocyte potentiating factor [22-25].

The most frequently evaluated immunohistochemical marker was EMA followed by BER-EP4, CEA and calretinin. Among the serum markers, the most frequently investigated were SMRP and CEA, and among effusion markers CEA, CA15-3, HA and SMRP [20]. The most valuable tissue markers for mesothelial cells are calretinin, EMA and WT-1 as positive markers and CEA, Ber-EP4 and TTF-1 (thyroid transcription factor 1) as negative markers. The most valuable tissue markers to discriminate mesothelioma cells from other malignant tumor cells appear to be CEA, Ber-EP4 and calretinin. The International Mesothelioma Panel recommends that at least 2 mesothelial and 2 carcinoma markers be used in addition to a pancytokeratin for a reliable diagnosis of MPM. None of these antibodies are $100 \%$ specific, and false positives (which often show $<10 \%$ stain- 
ing) can occur in either direction. One exception is the carcinoma marker TTF-1, for which no case of mesothelioma with a positive staining has been published [26]. In discriminating mesothelioma from non-malignant diseases, EMA and SMRP are considered the most valuable markers, but none of the markers had sufficient discriminatory value to differentiate mesothelioma from other diseases.

Among a large panel of markers, the MUC1 antibody (clone E29 of EMA), one of the most valuable and promising markers of which the principal isoform MUC1 or MUC1-TM is expressed at the epithelial surface in various tissues, resulted in the best sensitivity $(\sim 80 \%)$ with no false positive in MPM. The gene product MUC1 is even more important because it can be detected in the circulation and its presence (a specific glycolsylated form) could result in targeted therapies [Theratope, STn, $\operatorname{sialyl}(\mathrm{Tn})][27,28]$.

GLUT-1 is a new promising marker in the distinction of malignant mesothelial lesions. Upregulation of glucose transport across the plasma membrane is mediated by a family of glucose transporter proteins named GLUT. Overexpression of the hypoxia-responsive GLUT-1 has been frequently observed in several carcinomas and is a very important limiting factor in the transport and metabolism of glucose in malignant cells. In contrast to malignant tumors, including mesothelioma, GLUT-1 expression has been relatively undetectable in normal tissue and benign epithelial tumors. A recent study by Kato et al. [29] showed that GLUT-1 reactivity was found in 40 of 40 mesotheliomas; whereas all 40 cases of reactive mesothelial hyperplasia were negative. Thus, GLUT-1-positive staining is a helpful marker for MPM - both epithelioid and sarcomatoid.

IMP3 is an oncofetal protein involved in embryogenesis and it promotes tumor cell proliferation, invasion and metastasis. Studies have shown that IMP3 is an important cancer-specific protein that is associated with aggressive and advanced cancers, and it is specifically expressed in malignant tumors, but it is not found in benign tissue. Shi et al. [30] recently showed that IMP3 was negative in all 64 reactive mesothelial lesions tested while it was positive in 33 of 45 (73\%) malignant mesotheliomas leading to the conclusion that staining for IMP3 can be a useful immunohistochemical marker in the distinction of malignant mesothelioma from reactive mesothelial cell proliferations. In order to study promising markers, prospective accuracy studies are necessary; retrospective case-control studies could be also useful [31-33].
The added value of these markers to established diagnostic criteria such as patient characteristics and previous clinical tests should be investigated $[33,34]$.

Currently, the value of non-invasive markers in the diagnosis of mesothelioma remains to be determined since studies addressing this objective were of limited value and results were inconclusive due to considerable variability among the studies.

\section{Prognostic Value}

Biomarkers may help in the prognosis of MPM. A significant negative prognostic marker in MPM patients is SMRP [35], but further studies are needed. Recent studies have shown that loss of p16 is associated with poor survival $[36,37]$.

Angiogenesis seems to be very important in MPM progression. In fact, increased microvessel density has been associated with poor survival [38], and proteins involved in regulating angiogenesis have been implicated in the prognosis of MPM. The engine of angiogenesis is hypoxia. A reduced level of BAX, a tumor suppressor gene downregulated by tumor hypoxia, has been associated with a poor outcome [39]. Elevated levels of VEGF in pleural effusion are associated with diminished survival in MPM patients [6], and VEGF overexpression monitored by immunohistochemistry independently predicts poor survival in MPM patients $(\mathrm{p}=0.0002)$ [40]. High levels of VEGF and fibroblast growth factor (FGF) 2, or co-expression of TGF- $\beta$, VEGF, FGF1 and FGF2 are also associated with a poor outcome [41]. PTEN expression was found as a strong predictor of survival in 126 mesothelioma patients [42].

A four-gene signature comprising KIAA097, GDIA1 (GDP-dissociation inhibitor 1), CTHBP (cytosolic thyroid hormone binding protein) and an expressed sequence tag similar to the L6 tumor antigen (which correctly classified a training sample into good and poor prognostic groups) predicted the correct outcome in a significant number of cases, encouraging future research on novel prognostic molecular markers to diagnose/ monitor disease and assess treatment response [43]. The presence of an 11-gene, oncogene-driven pathway signature, correlated with a stem-cell-like expression profile, is associated with a poor prognosis in patients with MPM [44]. In the same way, a large gene expression analysis identified and validated aurora kinases as predictors of outcome.

In fact, mitosis or proliferation, diploidy and S-phase fraction were identified as significant indices, and expression of regulators of mitosis and cell cycle control was 
increased in more aggressive cancers [36]. Pass et al. [45] investigated whether specific microRNAs could help to distinguish patients with surgically treated mesothelioma into those with a good or bad prognosis.

By regulating cell functions such as growth, survival, motility/migration and invasion, the c-mesenchymal-epithelial transition (c-MET) receptor tyrosine kinase/hepatocyte growth factor axis also accounts for a critical pathway in MPM. A recent study showed that, disregarding the intracellular c-MET receptor traffic, only c-MET plasma membrane localization could be a relevant prognostic biomarker in MPM [46].

\section{Predictive Value}

There are no established indicators of responsiveness that can be used to optimize treatment in MPM. For targeted therapy, however, low VEGF serum levels may be useful in predicting the response to treatment with bevacizumab, and for chemotherapy, few studies have identified predictors of response to pemetrexed and/or cisplatin/carboplatin treatment in patients with MPM. It is hypothesized that low ERCC-1 expression might predict increased sensitivity to platinum-based chemotherapy, possibly due to the saturation of the enzyme complex. Conversely, high ERCC-1 levels may predict resistance to platinum-based chemotherapy.

Thymidylate synthase (TS) mRNA expression levels were inversely correlated with pemetrexed activity in different tumor cells, whereas other studies suggested a correlation between high levels of TS protein expression and reduced sensitivity to pemetrexed in colon and lung cancer cells $[47,48]$. Furthermore, TS mRNA and protein expression were predictive of treatment response in patients with advanced breast cancer treated with pemetrexed alone and in patients with non-small cell lung cancer treated with pemetrexed/gemcitabine neoadjuvant therapy, respectively [49]. In a recent retrospective analysis, Righi et al. [50] observed that low TS protein levels are predictive of improved time to progression and overall survival (OS). Another retrospective analysis of TS and ERCC-1 protein expression by immunohistochemistry in 99 MPM patients treated with the carboplatin/pemetrexed regimen found that TS expression was a predictor of clinical outcome [51].

Retrospective studies on candidate predictive biomarkers in MPM specimens may provide a strong rationale for future trials. However, to be able to identify the best biomarkers for personalized MPM chemotherapy in prospective trials, optimization and standardization of methodologies are required, as well as the use of large and uniformly treated cohorts and the incorporation of both emerging candidate biomarkers and genotype studies. Probably, considering the rarity of this disease, the creation of a collaborative network which would allow analyzing the role of several biomarkers in an appropriate number of uniformly treated patients with MPM may be a good strategy. Surely, the detection of novel biomarkers will be one of the major targets of MPM research in the future.

\section{To the Bedside: New Treatment Approaches}

The role of surgery and radiotherapy remains controversial in MPM and should be further explored. Platinum-based combination chemotherapy with antimetabolites (pemetrexed/raltitrexed) became the cornerstone of first-line treatment $[52,53]$ during the last decade, but results were of limited value with respect to patient survival. Issued from an increasing knowledge on MPM pathogenesis, targeted agents and novel therapeutic strategies under investigation in preclinical models and clinical trials are presented below.

\section{Epithelial Growth Factor Receptor}

The epithelial growth factor receptor (EGFR) plays a role in cell proliferation, differentiation, migration, adhesion and survival [54], and it is overexpressed at both protein and transcriptional levels in $>50 \%$ of MPM patients [55].

The Cancer and Leukemia Group B (CALGB) 30101 phase II trial enrolled 43 chemotherapy-naïve patients with MPM, and all patients received $500 \mathrm{mg}$ gefitinib once a day until disease progression or unacceptable toxicity. The results showed a 3-month progression-free survival (PFS) of $40 \%$, and this was compared with a similar historic control group of 337 chemotherapy-naïve MPM patients from the CALGB database [56]. This was higher than that seen in the gefitinib trial (40\% PFS at 3 months), and the authors therefore concluded that single-agent gefitinib was not active in malignant mesothelioma [57]. In the second phase II trial, 63 chemotherapy-naïve patients with advanced or recurrent MPM and a performance status of $0-1$ were treated with erlotinib. There were no responses, although 14 of 33 (42\%) patients had stable disease (SD) [58], and the authors therefore concluded that single-agent erlotinib was not effective in MPM.

One explanation for the low efficacy of EGFR inhibitors despite EGFR overexpression might be the rarity of EGFR mutations in mesothelioma [59]. Some research 
groups reported that there was no relationship between EGFR overexpression and outcome in patients with MPM $[55,60,61]$, and others have reported that EGFR overexpression is associated with improved outcome [62-64]. However, EGFR overexpression in MPM is seen more commonly in the epithelial histological subtype, which is associated with improved patient survival, but it is not an independent prognostic marker [63, 64].

There is significant cross-talk between the EGFR pathway and other receptor signaling pathways. Downstream proteins within the EGFR signaling pathway, such as PI3K and AKT, are utilized by other tyrosine kinase receptor growth factor pathways, including the c-MET and IGF-1 receptor pathways [65-67]. Immunohistochemically, c-MET protein was found to be overexpressed in MPM and in samples of normal pleura. In mesothelioma cell lines, treatment with the small molecule c-MET inhibitor SU11274 resulted in dose-dependent growth inhibition [68]. The IGF-1 receptor has been shown to be important in the malignant phenotype of MPM101 and treatment of mesothelioma cell lines with the IGF-1 receptor inhibitors NVP-AEW541 and AG1024 resulted in dose-dependent growth inhibition [69]. AG1024 was also shown to enhance the cytotoxic effect of cisplatin in mesothelioma cells [70].

Significant cross-talk exists between EGFR and cyclooxygenase 2 (COX2) [71]. COX2, which is overexpressed in many solid tumors, is also a potential therapeutic target [72-74]. In MPM, immunohistochemical analysis of the COX 2 protein revealed overexpression in $59-100 \%$ of tumor samples [75-77]. Treatment of mesothelioma cell lines with COX 2 inhibitors induced cy totoxicity and also enhanced the effects of pemetrexed [78, 79].

\section{K-ras, BRAF and PI3KCA Mutations}

Screening for K-ras gene mutations at codons 12, 13 and 61 in several mesothelioma cell lines did not reveal any mutation $[80,81]$, which was in agreement with a study by $\mathrm{Ni}$ et al. [82] in 17 mesothelioma samples.

In MPM, BRAF gene mutations were absent in 53 tumors and in 6 tumor cell lines [83]. Finally, Suzuki et al. [84] studied 21 mesothelioma cell lines and did not find any PI3KCA gene mutation.

\section{PTEN}

PTEN gene deletion in 1 of 9 mesothelioma cells lines and loss of PTEN protein expression in 2 of 26 mesothelioma samples, both resulting in elevated AKT activity, were reported [85]. In an immunohistochemical study of 19 MPM tissue samples for AKT pathway-related pro- teins, PTEN protein expression was absent in $16 \%$ and mild-to-moderate PTEN expression was found in $86 \%$. Suzuki et al. [84] reported loss of PTEN protein expression in 2 and low expression in 11of 21 mesothelioma cell lines, resulting in constitutive activation of AKT. In a larger series of 206 MPM tissue samples, PTEN protein expression was lost in $62 \%$ of the cases (score 0 ) whereas $14 \%$ had weak (score 1), 10\% moderate (score 2) and $14 \%$ strong PTEN expression. Loss of PTEN protein expression can be considered an independent poor prognostic biomarker in 126 of 206 MPM patients. Median survival was significantly longer in patients with PTEN-positive tumors than in those with PTEN-negative tumors [42]. Loss of the PTEN protein, resulting in constitutive activation of $\mathrm{AKT}$, may induce resistance to both EGFR tyrosine kinase inhibitors and anti-EGFR monoclonal antibodies as they act upstream of PTEN.

\section{VEGF/VEGF Receptors}

Preclinical studies have shown that VEGF and VEGF receptors (VEGFR) are expressed in MPM, and circulating VEGF levels are higher in malignant mesothelioma (MM) patients than in healthy individuals or in patients with other malignancies [86]. Increased VEGF levels are positively correlated with microvascular density and associated with a poor prognosis [87]. VEGF levels increased with MPM progression [88]. VEGF stimulates MPM cells in a dose-dependent manner, and MPM cell growth was found to be inhibited by anti-VEGF antibodies [6].

A phase II trial combining erlotinib and bevacizumab reported that $50 \%$ of patients had SD; median PFS was 2.2 months and median OS was 5.8 months [89].

Development of hypertension is reported as a possible surrogate marker for bevacizumab activity and a potential significant predictor of outcome.

One phase II trial evaluating vatalanib in previously untreated patients showed no correlation between baseline VEGF or PDGF levels and response, PFS or survival [90].

Cediranib also seems to be a potent pan-VEGFR inhibitor that has antitumor activity in several solid tumors [91-93]. One phase II trial by Garland et al. [94] included 54 patients with MPM who had received prior treatment with platinum-based chemotherapy; a partial response (PR) was noted in $9 \%$ of patients, SD in 33\%, with a median PFS of 2 months and a median OS of 10 months.

Semaxanib is an inhibitor of the VEGF-1 receptor and, less potently, the PDGF receptor (PDGFR) and c-kit; due 
to the low oral bioavailability of this molecule, intravenous administration is required [95].

A phase I trial tested thalidomide in MPM patients, including $33 \%$ of chemotherapy-naïve patients. There was no PR or complete response; $27.5 \%$ of patients were free of progression after 6 months, and OS was 7.6 months [96]. Its use as maintenance therapy following cytotoxic chemotherapy showed no evidence of improvement in either PFS or OS

In patients with unresectable mesothelioma, sorafenib had limited activity, which supported the results with other VEGFR tyrosine kinase inhibitors [97]. In a phase I study, sorafenib (400 mg b.i.d.) was combined with doxorubicin $\left(60 \mathrm{mg} / \mathrm{m}^{2}\right)$, and this combination was well tolerated. The increased doxorubicin exposure with sorafenib did not result in significantly increased toxicity. These results justify further clinical investigation [98].

Sunitinib was tested in a phase II trial in MPM as second-line treatment after chemotherapy with platinum and antimetabolites but did not reach the main objective of the study with the following results: PR 12\%; SD 65\%; median time to progression of 3.5 months and OS of 7 months (Nowak et al., IMIG 2011, unpubl. data).

High levels of VEGF and FGF2 or co-expression of TGF, VEGF, FGF1 and FGF2 have been found to be associated with a poor outcome [99]. MPM exhibits high expression levels of the surrogate marker of hypoxia, hypoxia-inducible factor 1 [100].

Imatinib is a highly selective inhibitor of the bcr/abl mutated tyrosine kinase, as well as of both c-kit and PDGFRs. Several phase II studies have been conducted with imatinib mesylate in MPM refractory to chemotherapy or chemo-naïve patients, but negative results were reported [101-103]. In vitro and in vivo experiments demonstrated that STI-571 can cause MPM cell apoptosis and death through inhibition of the AKT/PI3K pathway and that it can also enhance MPM sensitivity to gemcitabine or pemetrexed [104]. Patients with MPM are currently being enrolled in a phase I study of imatinib combined with cisplatin and pemetrexed [105].

\section{PDGF/PDGFR}

PDGF $\alpha \mathrm{R}$ is overexpressed in mesothelioma cells, and increased secretion of PDGF is thought to cause thrombocytemia, which is considered a prognostic factor of adverse events and occurs in many patients with MPM [106]. Indeed, high serum PDGF levels in MPM patients seem to be an independent predictor of poor survival [107].

Overexpression of PDGF $\alpha$ has been shown in MPM cell lines, and blocking PDGFR has led to growth inhibi- tion in vitro [108]. Combined with the fact that c-kit expression is seen in $26 \%$ of MPM patients, this spurred clinical trials investigating imatinib in MM [109].

Inhibition of PDGFR with imatinib and paclitaxel has been shown to lower interstitial fluid pressure with a possible subsequent improvement in drug delivery and increased efficacy in vitro [110]. In a phase I trial with imatinib in combination with gemcitabine including $5 \mathrm{MM}$ patients, 1 patient had PR [111]. In preclinical trials, dasatinib had cytotoxic effects and resulted in decreased migration and invasion in mesothelioma cell lines [112]. In another phase II trial conducted in 46 inoperable patients with no responders, PFS was 2 months and median OS 4.8 months [113].

\section{PI3K/AKT/mTOR Pathway}

Sirolimus is approved as an immunosuppressant used especially in kidney transplants, and it has an antiproliferative effect on the PI3K/AKT/mTOR (mammalian target of rapamycin) pathway through the tyrosine kinase mTOR. The PI3K/AKT/mTOR pathway is often aberrant in MPM, and in vitro studies have shown that inhibition of the pathway may induce apoptosis in MPM cell lines $[85,114]$. Temsirolimus, a derivative of rapamycin, has been evaluated in a phase I trial including $2 \mathrm{MM}$ patients. None responded to the treatment [115]. In an in vitro study, combination therapy with cisplatin and sirolimus had synergistic antitumor effects in MPM cell lines [116].

\section{Mesothelin}

Mesothelin is highly expressed in several cancers, including ovarian cancer, pancreatic cancer, some squamous cell carcinomas and mesotheliomas (epithelioid subtype cells only) $[117,118]$. The high membrane expression of mesothelin in MM and the limited distribution of mesothelin in normal tissue raised interest for mesothelin as an antitumor target [119]. A phase I trial has been conducted, but none of the MM patients showed a response [120].

Several agents presenting activity in preclinical models are being developed to target mesothelin: a recombinant immunotoxin (SS1P), a humanized monoclonal antibody (MORAb-009) and an attenuated Listeria vector that encodes human mesothelin (CRS-207). SS1P and MORAb-009 have completed phase I evaluation. For SS1P, 4 of the 33 evaluable patients treated had minor responses, 19 had SD, while 10 had progressive disease [121]. The result of this study and previously reported synergistic effects of SSP1 in combination with chemotherapy [122] triggered an ongoing phase II study. 


\section{Ribonuclease}

Ranpirnase is a ribonuclease that degrades RNA, and the irreparable RNA damage may constitute a death signal for apoptosis and also contributes to the inhibition of cell growth and proliferation. For this reason, ranpirnase has been tested in a phase II trial and $4.9 \%$ of patients responded to therapy; OS was 6 months. Several patients were excluded from the study due to adverse events, e.g. renal insufficiency, allergic reactions, arthralgia and peripheral edema [123]. Whether this small advantage is of enough clinical value to continue further research with this drug remains presently unclear.

\section{Asparagine-Glycine-Arginine-Human TNF- $\alpha$}

TNF- $\alpha$ has antitumor activity through activation of apoptosis. However, TNF treatment is associated with severe toxicities, which only allow TNF to be administered in doses at least tenfold lower than the dose effective in preclinical models $[124,125]$. NGR-human TNF consists of human TNF- $\alpha$ fused to the tumor-homing peptide asparagine-glycine-arginine (NGR) able to selectively bind an aminopeptidase $\mathrm{N}$-isoform overexpressed in tumor blood vessels. A phase II trial by Gregorc et al. [126] evaluating NGR-human TNF included 57 patients: PR was seen in $1(2 \%)$ patient. Eighteen (31\%) patients with SD had a median PFS of 4.4 months. Overall, PFS and OS were 2.8 and 12.1 months, respectively. Treatment was well tolerated.

\section{HDACi}

Histone proteins exist in either acetylated or deacetylated configurations, and the equilibrium between the two forms is regulated by histone acetyltransferase and HDAC. Inhibition of histone acetylation results in acetylation of histone proteins and expression of genes associated with cell cycle arrest, apoptosis and tumor suppression. Moreover, HDACis induce acetylation of nonhistone proteins leading to other anticancer effects such as inhibition of angiogenesis, motility and invasion of tumor cells. Many specific or pan-HDACis have been tested in MPM, including suberoylanilide hydroxamic acid (SAHA/vorinostat), panobinostat or valproic acid (VPA), either alone or in combination with chemotherapy. Vorinostat has already gained FDA approval for the treatment of cutaneous T-cell lymphoma [13].

Preliminary evidence from a phase I trial suggested that vorinostat might exert clinically meaningful activity in patients with mesothelioma [127]. However, a phase III trial (Vantage 014) comparing vorinostat with placebo in 660 pretreated mesothelioma patients who had progressed on one or two prior therapies failed to demonstrate a significant improvement in OS [128]. Vorinostat or placebo was given 3 days per week during a 21-day cycle. Although the median PFS for vorinostat was 6.3 versus 6.1 weeks, this did not translate into a statistically significant OS benefit for vorinostat compared with placebo. In a phase I trial by Ramalingam et al. [129], combination treatment with vorinostat, carboplatin and paclitaxel led to SD in the 1 MPM patient included. The same team evaluated another HDACi, belinostat, in a phase II trial in 13 patients. There were no responders, and PFS was only 1 month and OS was 5 months. Only 2 patients (15\%) had SD [130]. In vitro studies suggest increased efficacy of HDACi in combination with other agents [131].

In vitro data suggest that VPA has proapoptotic effects in MPM, and synergistic effects of VPA with chemotherapeutic agents such as doxorubicin were noted. In a recent phase II study by Scherpereel et al. [132], the effect and safety of VPA combined with doxorubicin were evaluated in a total of 45 MPM patients with performance status 0-2 after at least 1 chemotherapy (platinium/pemetrexed). Response rate was $16 \%$ (95\% CI 3-25\%), and disease control rate was 36\% (95\% CI 22-51\%). OS was 6.7 months (95\% CI 4.9-8.5 months). New clinical trials testing HDAC (vorinostat or VPA) combined with cisplatin and pemetrexed are now proposed for chemonaïve MPM patients.

\section{CBP501 EIMC-A12}

Since most cancer cells are dependent on the G2 checkpoint to survive, this has led to the development of CBP501, a G2 checkpoint abrogator. One phase I trial by Shapiro et al. [133] included 3 patients treated with CGP501 combined with cisplatin. One patient had PR and PFS of 9.7 months. Two patients had SD that lasted 11 and 3 months, respectively. A combined phase I/II trial is currently ongoing and enrolling patients with solid tumors (phase I) and MPM patients (phase II). MPM patients will be randomized to pemetrexed and cisplatin with or without CBP501.

IMC-A12 is an antibody targeting IGF-1. Inhibition of the IGF-1 receptor has led to decreased cell proliferation and enhanced the cytotoxic effect of cisplatin in vitro [70].

\section{Immunotherapy and Gene Therapy}

Immunotherapy with systemically administered IL-2 has limited efficacy but substantial adverse effects [134, 135] whereas intrapleural administration of IL-2, which is well tolerated and results in objective responses, needs 
further study to assess its superiority to conventional treatment [136]. Nevertheless, further studies of systemic IL-2, as well as artificial upregulation of endogenous IL-2 by gene transfer, are underway, based upon data from murine models of mesothelioma [137].

Rapamycin, a natural macrolide approved as immunosuppressor, was found to exert antiproliferative effects by inhibition of serine/threonine kinase, which is called mTOR in mammals. Synthetic derivatives or 'rapalogs' have been developed to improve the pharmacological properties of rapamycin: everolimus, temsirolimus and deforolimus.

Bortezomib is a potent inhibitor of the $20 \mathrm{~S}$ proteasome, which has shown cytotoxic effects in vitro and in MM xenografts in vivo $[138,139]$. On the basis of promising preclinical data, two phase II trials of bortezomib have been initiated in Europe. One trial is exploring single-agent activity in the second-line setting and in patients with a performance status of 2 in the first-line setting [140].

Interferon (IFN)- $\alpha 2 a$ and IFN- $\gamma 1 b$ have been combined with various standard chemotherapies for mesothelioma. The response rates in these trials have been variable (for IFN- $\alpha$ 2b plus cisplatin, 27-36\% [141]; for IFN- $\alpha 2 b$ with doxorubicin alone, $11 \%$ [142] and for IFN$\alpha 2 \mathrm{~b}$ with cisplatin plus doxorubicin, 29\% [143]).

High-dose methotrexate was combined with IFN- $\alpha$ and IFN- $\gamma$ in a series of 24 evaluable patients with advanced mesothelioma. Seven (29\%) had a PR, and the 1and 2-year survival rates were 62 and $31 \%$, respectively [144]. A small study of epirubicin plus IL-2 showed only $1 \mathrm{PR}$ among 25 patients with advanced disease [145].

Vaccine approaches are also under investigation for the treatment of MPM. WT-1 peptide epitopes that stimulate T-cell immunity are currently under evaluation for the treatment of mesothelioma. Preliminary results from ongoing studies have documented the safety of this vaccine.

Interesting preliminary results were observed after administration of Mycobacterium vaccae in a limited number of patients. This needs to be confirmed before exploring this treatment further.

Exploiting the immunostimulatory capacities of dendritic cells (DCs) holds great promise for cancer immunotherapy. Currently, DC-based immunotherapy is evaluated clinically in a number of malignancies, including melanoma and urogenital and lung cancer, showing variable but promising results. In a murine model, Hegmans et al. [146] demonstrated that immunotherapy using pulsed DCs may emerge as a powerful tool to control mesothelioma outgrowth. This team showed that mesothe- lioma is infiltrated by immune effector cells but also contains cytokines and regulatory $\mathrm{T}$ cells that suppress an efficient immune response.

Immunotherapy of mesothelioma might be more effective when combined with drugs that eliminate or control regulatory T cells [147]. The results of a phase I clinical trial addressing the safety and feasibility of tumor lysate- or exosome-pulsed DCs to induce tumor-specific cytotoxic T-lymphocyte responses in patients with MPM were recently published [148]. The goal of this trial in 10 MPM patients was to assess the safety and immunological response induced by the intradermal and intravenous administration of tumor lysate-pulsed DCs at 2-week intervals after chemotherapy. The treatment was safe with no grade 3 or 4 toxicities associated with the vaccines or any evidence of autoimmunity; moderate fever was the only side effect. Interestingly, local accumulation of infiltrating $\mathrm{T}$ cells was found at the site of vaccination. Immunological response to tumor cells was detected in a subgroup of mesothelioma patients.

\section{Intracavitary Therapy}

The pleural space provides an easy access for molecules to the body and novel intrapleural therapies for mesothelioma are under clinical development [149]. Intraoperative intracavitary chemotherapy has been used in an effort to improve local disease control. In a series of 92 patients, hyperthermic intracavitary perfusion with cisplatin $\left(225 \mathrm{mg} / \mathrm{m}^{2}\right)$ was performed following extrapleural pneumonectomy [150]. Recurrence of pleural mesothelioma was seen in $51 \%$ of the patients, but the value of this approach remains uncertain due to selection bias and the lack of randomized trials.

Intrapleural instillation of a replication-deficient, recombinant adenovirus has been used to insert the gene for the herpes simplex virus thymidine kinase gene, thus making tumor cells sensitive to the normally nontoxic antiviral drug ganciclovir or to deliver the gene for human type I IFNs $(\beta / \alpha)$ to induce antitumor immune responses [151-153]. A number of long-term clinical responses suggest that this approach has some promise, and also has the capacity to induce significant humoral and cellular antitumor immune responses $[153,154]$.

One of the most attractive targets for therapy is mesothelin, a tumor differentiation antigen that is overexpressed by most epithelial mesotheliomas but not by normal cells [155]. Three mesothelin-targeted agents are in various stages of clinical development. These include SS1P (anti-mesothelin dsFv-PE38), a recombinant immunotoxin composed of an anti-mesothelin $\mathrm{Fv}$ fragment 
linked to a truncated Pseudomonas exotoxin [156-158], MORAb-009, a chimeric anti-mesothelin monoclonal antibody [159], and CRS-207, a live-attenuated Listeria monocytogenes vector encoding human mesothelin [160]. The rationale for mesothelin as a tumor vaccine is that mesothelin elicits a strong T-cell response in patients [161]. Phase I trials have been completed with SS1P and MORAb-009, and some evidence of minor antitumor activity has been observed with SS1P [162].

\section{Conclusion}

It is obvious that a continuous collaboration between clinicians, pathologists [163] and basic researchers will be a crucial step to improve the treatment of mesothelioma patients. Thus, during these last 5 years, many studies on VEGF/VEGFR, PI3K/AKT/mTOR pathway, or on meso- thelin, HDACi and immunotherapy/gene therapy from various international teams have brought new advances from the bench to the bedside that are very encouraging. However, further in-depth analysis is required for a more advanced deciphering of the step-by-step process leading from early increased mesothelial cell proliferation to invasive mesothelioma, from which we are expecting the development of definitively effective therapy. Moreover, many questions remain to be answered such as: how long should we give first-line treatment? Which second-line treatment should we use? What is the role of targeted therapies, either alone or combined with chemotherapy, surgery and/or radiotherapy? Therefore, as already emphasized by all mesothelioma experts, it is essential that all MPM patients should be recruited in clinical trials and research studies to fasten translational research and to improve the management of this rare and aggressive cancer [164].

\section{References}

1 Yang H, Bocchetta M, Kroczynska B, et al: TNF- $\alpha$ inhibits asbestos-induced cytotoxicity via a NF- $\mathrm{KB}$-dependent pathway, a possible mechanism for asbestos-induced oncogenesis. Proc Natl Acad Sci USA 2006;103: 10397-10402.

$>2$ Zucali PA, Ceresoli GL, De Vincenzo, et al: Advances in the biology of malignant pleural mesothelioma. Cancer Treat Rev 2011;37: 543-558.

$>3 \mathrm{Xu}$ A, Wu LJ, Santella RM, et al: Role of oxyradicals in mutagenicity and DNA damage induced by crocidolite asbestos in mammalian cells. Cancer Res 1999;59:5922-5926.

4 Ramos-Nino ME, Timblin CR, Mossman BT: Mesothelial cell transformation requires increased AP-1 binding activity and ERKdependent Fra-1 expression. Cancer Res 2002;62:6065-6069.

$>5$ Liu Z, Klominek J: Chemotaxis and chemokinesis of malignant cells to multiple growths factors. Anticancer Res 2004;24:1625-16230.

6 Strizzi L, Catalano A, Vianale G, et al: Vascular endothelial growth factor is an autocrine growth factor in human malignant mesothelioma. J Pathol 2001;193:468-475.

$>7$ Galffy G, Mohammed KA, Downling PA, et al: Interleukin 8: an autocrine growth factor for malignant mesothelioma. Cancer Res 1999;59:367-371.

$>8$ Carbone M, Kratzke RA, Testa JR: The pathogenesis of mesothelioma. Semin Oncol 2002;29:2-17.

>9 Murthy SS, Testa JR: Asbestos, chromosomal deletions, and tumor suppressor gene alterations in humal malignant mesothelioma. J Cell Physiol 1999;180:150-157.
0 Baylin SB, Ohm JE: Epigenetic gene silencing in cancer - a mechanism for early oncogenic pathway addiction? Nat Rev Cancer 2006;2: 107-116.

11 Bolden JE, Peart MJ, Johnstone RW: Anticancer activities of histone deacetylase inhibitors. Nat Rev Drug Discov 2006;5:769784.

12 Cao XX, Mohuiddin I, Ece F, et al: Histone deacetylase inhibitor downregulation of bcl$\mathrm{xl}$ gene expression leads to apoptotic cell death in mesothelioma. Am J Respir Cell Mol Biol 2001;25:562-568.

13 Frew AJ, Johnstone RW, Bolden JE: Enhancing the apoptotic and therapeutic effects of HDAC inhibitors. Cancer Lett 2009;280: 125-133.

14 Cheng JQ, Lee WC, Klein MA, et al: Frequent mutations of NF2 and allellic loss from chromosome band 22q12 in malignant mesothelioma: evidence for a two-hit mechanism of NF2 inactivation. Genes Chromosomes Cancer 1999;24:238-242.

15 Poulikakos PI, Xiao GH, Gallagher R, et al: Re-expression of the tumor-suppressor NF2/ merlin inhibits invasiveness in mesothelioma cells and negatively regulates FAK. Oncogene 2006;25:5960-5968.

16 Thurneysen C, Opitz I, Kurtz S, et al: Functional inactivation of NF2/merlin in human mesothelioma. Lung Cancer 2009;64:140147.

$>17$ Fleury-Feith J, Lecomte C, Renier A, et al: Hemizygosity of Nf2 is associated with increased susceptibility to asbestos-induced peritoneal tumours. Oncogene 2003;22: 3799-3805.
18 Deguen B, Goutebroze L, Giovannini M, et al: Heterogeneity of mesothelioma cell lines as defined by altered genomic structure and expression of the NF2 gene. Int J Cancer 1998;77:554-560.

19 Lopez-Lago MA, Okada T, Murillo MM, et al: Loss of the tumor suppressor gene NF2, encoding merlin, constitutively activates integrin-dependent mTORC1 signaling. Mol Cell Biol 2009;64:140-147.

20 van der Bij S, Schaake E, Koffijberg H, et al: Markers for the non-invasive diagnosis of mesothelioma: a systematic review. Br J Cancer 2011;104:1325-1333.

21 Lyons-Boudreaux V, Mody DR, Zhai J, et al: Cytologic malignancy versus benignancy: how useful are the 'newer' markers in body fluid cytology? Arch Pathol Lab Med 2008; 132:23-28.

-22 Pass HI, Lott D, Lonardo F, et al: Asbestos exposure, pleural mesothelioma, and serum osteopontin levels. N Engl J Med 2005;353: 1564-1573.

23 Holloway AJ, Diyagama DS, Opeskin K, et al: A molecular diagnostic test for distinguishing lung adenocarcinoma from malignant mesothelioma using cells collected from pleural effusions. Clin Cancer Res 2006;12: 5129-5135.

24 Scherpereel A, Grigoriu B, Conti M, et al: Soluble mesothelin-related peptides in the diagnosis of malignant pleural mesothelioma. Am J Respir Crit Care Med 2006;173: 1155-1160. 
25 Creaney J, Yeoman D, Demelker Y, et al: Comparison of osteopontin, megakaryocyte potentiating factor, and mesothelin proteins as markers in the serum of patients with malignant mesothelioma. J Thorac Oncol 2008; 3:851-857.

-26 Husain AN, Colby TV, Ordóñez NG, et al: Guidelines for pathologic diagnosis of malignant mesothelioma: a consensus statement from the International Mesothelioma Interest Group. Arch Pathol Lab Med 2009; 133:1317-1331.

27 Miles D, Papazisis K: Rationale for the clinical development of STn-KLH (Theratope) and anti-MUC-1 vaccines in breast cancer (review). Clin Breast Cancer 2003;3(suppl 4):S134-S138.

-28 Wierecky J, Mueller M, Brossart P: Dendritic cell-based cancer immunotherapy targeting MUC-1. Cancer Immunol Immunother 2006;55:63-67.

29 Kato Y, Tsuta K, Seki K, et al: Immunohistochemical detection of GLUT-1 can discriminate between reactive mesothelium and malignant mesothelioma. Mod Pathol 2007;20: 215-220.

-30 Shi M, Fraire AE, Chu P, et al: Oncofetal protein IMP3, a new diagnostic biomarker to distinguish malignant mesothelioma from reactive mesothelial proliferation. Am J Surg Pathol 2011;35:878-882.

- 31 Biesheuvel CJ, Vergouwe Y, Oudega R, et al: Advantages of the nested case-control design in diagnostic research. BMC Med Res Methodol 2008;8:48.

- 32 Rutjes AW, Reitsma JB, Vandenbroucke JP, et al: Case-control and two-gate designs in diagnostic accuracy studies. Clin Chem 2005; 51:1335-1341.

-33 Moons KG: Criteria for scientific evaluation of novel markers: a perspective. Clin Chem 2010;56:537-541.

-34 Riley RD, Sauerbrei W, Altman DG: Prognostic markers in cancer: the evolution of evidence from single studies to meta-analysis, and beyond. Br J Cancer 2009;100:1219-1229.

- 35 Cristaudo A, Foddis R, Vivaldi A, et al: Clinical significance of serum mesothelin in patients with mesothelioma and lung cancer. Clin Cancer Res 2007; 17:5076-5081.

- 36 López-Ríos F, Chuai S, Flores R, et al: Global gene expression profiling of pleural mesothelioma: overexpression of aurora kinases and P16/CDKN2A deletion as prognostic factors and critical evaluation of microarraybased prognostic prediction. Cancer Res 2006;66:2970-2979.

37 Borczuk AC, Taub RN, Hesdorffer M, et al: P16 loss and mitotic activity predict poor survival in patients with peritoneal malignant mesothelioma. Clin Cancer Res 2005; 50:189-198.

-38 Edwards JG, Swinson DE, Jones JL, et al: Tumor necrosis correlates with angiogenesis and is a predictor of poor prognosis in malignant mesothelioma. Chest 2003;124: 1916-1923.
39 Kokturk N, Firat P, Akay H, et al: Prognostic significance of $\mathrm{Bax}$ and Fas ligand in erionite and asbestos induced Turkish malignant pleural mesothelioma. Lung Cancer 2005; 50:189-198.

40 Demirag F, Unsal E, Yilmaz A, et al: Prognostic significance of vascular endothelial growth factor, tumor necrosis, and mitotic activity index in malignant pleural mesothelioma. Chest 2005;128:3382-3387.

41 Kumar-Sing S, Weyler J, Martin MJ, et al: Angiogenic cytokines in mesothelioma: a study of VEGF, FGF-1 and -2, and TGF beta expression. J Pathol 1999;189:72-78.

42 Opitz I, Soltermann A, Abaecherli M, et al: PTEN expression is a strong predictor of survival in mesothelioma patients. Eur J Cardiothorac Surg 2008;33:502-506.

43 Gordon GJ, Rockwell GN, Godfrey PA, et al: Validation of genomic-based prognostic tests in malignant pleural mesothelioma. Clin Cancer Res 2005;11:4406-4414.

44 Glinsky GV, Berezovska O, Glinskii AB, et al: Microarray analysis identifies a deathfrom-cancer signature predicting therapy failure in patients with multiple types of cancer. J Clin Invest 2005;115:1503-1521.

45 Pass H, Goparaju C, Ivanov S, et al: HsamiR29 $c^{*}$ is linked to the prognosis of malignant pleural mesothelioma. Cancer Res 2010;70:1916-1924.

46 Levallet G, Vaisse-Lesteven M, Le Stang N, et al: Plasma cell membrane localization of $\mathrm{c}$ MET predicts longer survival in patients with malignant mesothelioma: a series of 157 cases from the MESOPATH Group. J Thorac Oncol 2012;7:599-606.

47 Sigmond J, Backus HH, Wouters D, et al: Induction of resistance to the multitargeted antifolate pemetrexed (ALIMTA) in WiDr human colon cancer cells is associated with thymidylate synthase overexpression. Biochem Pharmacol 2003;66:431-438.

48 Ceppi P, Volante M, Ferrero A, et al: Thymidylate synthase expression in gastroenteropancreatic and pulmonary neuroendocrine tumors. Clin Cancer Res 2008;14:10591064.

49 Bepler G, Sommers KE, Cantor A, et al: Clinical efficacy and predictive molecular markers of neoadjuvant gemcitabine and pemetrexed in resectable non-small cell lung cancer. J Thorac Oncol 2008;3:1112-1118.

50 Righi L, Papotti MG, Ceppi P, et al: Thymidylate synthase but not excision repair crosscomplementation group 1 tumor expression predicts outcome in patients with malignant pleural mesothelioma treated with pemetrexed-based chemotherapy. J Clin Oncol 2010;28:1534-1539.

51 Zucali PA, Giovannetti E, Destro A, et al: Thymidylate synthase and excision repair cross-complementing group 1 as predictors of responsiveness in patients with malignant pleural mesothelioma treated with pemetrexed and carboplatin. Clin Cancer Res 2011;17:2581-2590.
52 Pinto C, Ardizzoni A, Betta PG, et al: Expert opinions of the First Italian Consensus Conference on the Management of Malignant Pleural Mesothelioma. Am J Clin Oncol 2011;34:99-109.

53 Scherpereel A, Astoul P, Baas P, et al: Guidelines of the European Respiratory Society and the European Society of Thoracic Surgeons for the management of malignant pleural mesothelioma. Eur Respir J 2010;35: 479-495.

54 Yarden Y: The EGFR family and its ligands in human cancer. Signalling mechanisms and therapeutic opportunities. Eur J Cancer 2001;37(suppl 4):S3-S8.

55 Destro A, Ceresoli GL, Falleni M, et al: EGFR overexpression in malignant pleural mesothelioma. An immunohistochemical and molecular study with clinico-pathological correlations. Lung Cancer 2006;51:207-215.

-56 Herndon JE, Green MR, Chahinian AP, et al Factors predictive of survival among $337 \mathrm{pa}-$ tients with mesothelioma treated between 1984 and 1994 by the Cancer and Leukemia Group B. Chest 1998;113:723-731.

57 Govindan R, Kratzke RA, Herndon JE 2nd, et al: Gefitinib in patients with malignant mesothelioma: a phase II study by the Cancer and Leukemia Group B. Clin Cancer Res 2005;11:2300-2304.

58 Garland LL, Rankin C, Gandara DR, et al: Phase II study of erlotinib in patients with malignant pleural mesothelioma: a Southwest Oncology Group Study. J Clin Oncol 2007;25:2406-2413.

59 Cortese JF, Gowda AL, Wali A, et al: Common EGFR mutations conferring sensitivity to gefitinib in lung adenocarcinoma are not prevalent in human malignant mesothelioma. Int J Cancer 2006;118:521-522.

60 Gaafar R, Bahnassy A, Abdelsalam I, et al: Tissue and serum EGFR as prognostic factors in malignant pleural mesothelioma. Lung Cancer 2010;70:43-50.

61 Okuda K, Sasaki H, Kawano O, et al: Epidermal growth factor receptor gene mutation, amplification and protein expression in malignant pleural mesothelioma. J Cancer Res Clin Oncol 2008;134:1105-1111.

62 O’Byrne KJ, Edwards JG, Waller DA: Clinico-pathological and biological prognostic factors in pleural malignant mesothelioma. Lung Cancer 2004;45(suppl 1):S45-S48.

63 Dazzi H, Hasleton PS, Thatcher N, et al: Malignant pleural mesothelioma and epidermal growth factor receptor (EGF-R). Relationship of EGF-R with histology and survival using fixed paraffin embedded tissue and the F4, monoclonal antibody. Br J Cancer 1990;61:924-926.

64 Edwards JG, Swinson DE, Jones JL, et al: EGFR expression: associations with outcome and clinicopathological variables in malignant pleural mesothelioma. Lung Cancer 2006;54:399-407. 
65 Kono SA, Marshall ME, Ware KE, et al: The fibroblast growth factor receptor signaling pathway as a mediator of intrinsic resistance to EGFR-specific tyrosine kinase inhibitors in non-small cell lung cancer. Drug Resist Updat 2009;12:95-102.

66 Eyzaguirre A, Buck E, Iwata K, et al: Mechanisms of resistance to EGFR tyrosine kinase inhibitors: implications for patient selection and drug combination strategies. Target Oncol 2008;3:235-243.

67 Engelman JA, Janne PA: Mechanisms of acquired resistance to epidermal growth factor receptor tyrosine kinase inhibitors in nonsmall cell lung cancer. Clin Cancer Res 2008; 14:2895-2899.

-68 Jagadeeswaran R, Ma PC, Seiwert TY, et al: Functional analysis of c-Met/hepatocyte growth factor pathway in malignant pleural mesothelioma. Cancer Res 2006;66:352-361.

69 Whitson BA, Jacobson BA, Frizelle S, et al: Effects of insulin-like growth factor-1 receptor inhibition in mesothelioma. Thoracic Surgery Directors Association Resident Research Award. Ann Thorac Surg 2006;82: 996-1001.

-70 Kai K, D'Costa S, Sills RC, et al: Inhibition of the insulin-like growth factor 1 receptor pathway enhances the antitumor effect of cisplatin in human malignant mesothelioma cell lines. Cancer Lett 2009;278:49-55.

-71 Dannenberg AJ, Lippman SM, Mann JR, et al: Cyclooxygenase-2 and epidermal growth factor receptor: pharmacologic targets for chemoprevention. J Clin Oncol 2005;23: 254-266.

72 Hull MA: Cyclooxygenase-2: how good is it as a target for cancer chemoprevention? Eur J Cancer 2005;41:1854-1863.

-73 Amir M, Agarwal HK: Role of COX-2 selective inhibitors for prevention and treatment of cancer. Pharmazie 2005;60:563-570.

-74 Gasparini G, Longo R, Sarmiento R, Morabito A: Inhibitors of cyclo-oxygenase 2: a new class of anticancer agents? Lancet Oncol 2003;4:605-615.

75 Baldi A, Santini D, Vasaturo F, et al: Prognostic significance of cyclooxygenase-2 (COX-2) and expression of cell cycle inhibitors p21 and p27 in human pleural malignant mesothelioma. Thorax 2004;59:428-433.

-76 Edwards JG, Faux SP, Plummer SM, et al: Cyclooxygenase- 2 expression is a novel prognostic factor in malignant mesothelioma. Clin Cancer Res 2002;8:1857-1862.

\$7 O’Kane SL, Cawkwell L, Campbell A, Lind MJ: Cyclooxygenase-2 expression predicts survival in malignant pleural mesothelioma. Eur J Cancer 2005;41:1645-1648.

78 Catalano A, Graciotti L, Rinaldi L, et al: Preclinical evaluation of the nonsteroidal antiinflammatory agent celecoxib on malignant mesothelioma chemoprevention. Int J Cancer 2004;109:322-328.

Malignant Pleural Mesothelioma
79 O'Kane SL, Eagle GL, Greenman J, et al: COX-2 specific inhibitors enhance the cytotoxic effects of pemetrexed in mesothelioma cell lines. Lung Cancer 2010;67:160-165.

-80 Kitamura F, Araki S, Tanigawa T, et al: Assessment of mutations of Ha- and Ki-ras oncogenes and the $\mathrm{p} 53$ suppressor gene in seven malignant mesothelioma patients exposed to asbestos-PCR-SSCP and sequencing analyses of paraffin-embedded primary tumors. Ind Health 1998;36:52-56.

81 Kitamura F, Araki S, Suzuki Y, et al: Assessment of the mutations of $\mathrm{p} 53$ suppressor gene and $\mathrm{Ha}$ - and Ki-ras oncogenes in malignant mesothelioma in relation to asbestos exposure: a study of 12 American patients. Ind Health 2002;40:175-181.

82 Ni Z, Liu Y, Keshava N, et al: Analysis of Kras and p53 mutations in mesotheliomas from humans and rats exposed to asbestos. Mutat Res 2000;468:87-92.

83 Dote H, Tsukuda K, Toyooka S, et al: Mutation analysis of the BRAF codon 599 in malignant pleural mesothelioma by enriched PCR-RFLP. Oncol Rep 2004;11:361-363.

84 Suzuki Y, Murakami H, Kawaguchi K, et al: Activation of the PI3K-AKT pathway in human malignant mesothelioma cells. Mol Med Rep 2009;2:181-188.

85 Altomare DA, You H, Xiao GH, et al: Human and mouse mesotheliomas exhibit elevated AKT/PKB activity, which can be targeted pharmacologically to inhibit tumor cell growth. Oncogene 2005;24:6080-6089.

86 Ohta Y, Shridhar V, Bright RK, et al: VEGF and VEGF type $C$ play an important role in angiogenesis and lymphangiogenesis in human malignant mesothelioma tumours. $\mathrm{Br} \mathrm{J}$ Cancer 1999;81:54-61.

87 Dowell J, Kindler H: Antiangiogenic therapies for mesothelioma. Hematol Oncol Clin North Am 2005;19:1137-1145.

88 Yasumitsu A, Tabata C, Tabata R, et al: Clinical significance of serum vascular endothelial growth factor in malignant pleural mesothelioma. J Thorac Oncol 2010;5:479-483.

89 Jackman DM, Kindler HL, Yeap BY, et al: Erlotinib plus bevacizumab in previously treated patients with malignant pleural mesothelioma. Cancer 2008;113:808-814.

90 Jahan T, Gu L, Kratzke R, et al: Vatalanib in malignant mesothelioma: a phase II trial by the Cancer and Leukemia Group B (CALGB 30107). Lung Cancer 2011, E-pub ahead of print.

91 Mitchell CL, O’Connor JP, Roberts C, et al: A two-part phase II study of cediranib in patients with advanced solid tumours: the effect of food on single-dose pharmacokinetics and an evaluation of safety, efficacy and imaging pharmacodynamics. Cancer Chemother Pharmacol 2011;68:631-641.

92 Drevs J, Siegert P, Medinger M, et al: Phase I clinical study of AZD2171, an oral vascular endothelial growth factor signaling inhibitor, in patients with advanced solid tumors. J Clin Oncol 2007;25:3045-3054.
$\$ 93$ Matulonis UA, Berlin S, Ivy P, Tyburski K, et al: Cediranib, an oral inhibitor of vascular endothelial growth factor receptor kinases, is an active drug in recurrent epithelial ovarian, fallopian tube, and peritoneal cancer. J Clin Oncol 2009;27:5601-5606.

94 Garland L, Chansky K, Wosniak A, et al: Phase II study of cediranib in patients with malignant pleural mesothelioma: SWOG S0509. J Thorac Oncol 2011;6:1938-1945.

95 Morabito A, De Maio E, Di Maio M, et al: Tyrosine kinase inhibitors of vascular endothelial growth factor receptors in clinical trials: current status and future directions. Oncologist 2006;11:753-764.

-96 Baas P, Boogerd W, Dalesio O, et al: Thalidomide in patients with malignant pleural mesothelioma. Lung Cancer 2005;48:291296.

97 Dubey S, Jänne PA, Krug L, et al: A phase II study of sorafenib in malignant mesothelioma: results of Cancer and Leukemia Group B 30307. J Thorac Oncol 2010;5: 1655-1661.

98 Richly H, Henning BF, Kupsch P, et al: Results of a phase I trial of sorafenib (BAY439006) in combination with doxorubicin in patients with refractory solid tumours. Ann Oncol 2006;17:866-873.

99 König JE, Tolnay E, Wiethege T, Müller KM: Co-expression of vascular endothelial growth factor and its receptor flt- 1 in malignant pleural mesothelioma. Respiration 2000;67:36-40.

100 Klabatsa A, Sheaff MT, Steele JP, et al: Expression and prognostic significance of hypoxia-inducible factor 1alpha (HIF-1 alpha) in malignant pleural mesothelioma (MPM). Lung Cancer 2006;51:53-59.

101 Millward M, Parnis F, Byrne M, et al: Phase II trial of imatinib mesylate in patients with advanced pleural mesothelioma (abstract 912). Proc Am Soc Clin Oncol 2003;22:912.

102 Villano J, Husain A, Stadler M, Hanson L, Vogelzang N, Kindler H, et al: A phase II trial of imatinib mesylate in patients (pts) with malignant mesothelioma (MM). J Clin Oncol 2004;22:14.

103 Mathy A, Baas P, Dalesio O, et al: Limited efficacy of imatinib mesylate in malignant mesothelioma: a phase II trial. Lung Cancer 2005;50:83-86.

104 Porta C, Mutti L, Tassi G: Negative results of an Italian Group for Mesothelioma (G.I.Me.) pilot study of single-agent imatinib mesylate in malignant pleural mesothelioma. Cancer Chemother Pharmacol 2007;59:149-150.

105 Bertino P, Porta C, Barbone D, et al: Preliminary data suggestive of a novel translational approach to mesothelioma treatment: imatinib mesylate with gemcitabine or pemetrexed. Thorax 2007;62:690-695. 
106 Edwards JG, Cox G, Andi A, et al: Angiogenesis is an independent prognostic factor in malignant mesothelioma. Br J Cancer 2001;85:863-868.

107 Filiberti R, Marroni P, Neri M, et al: Serum PDGF-AB in pleural mesothelioma. Tumour Biol 2005;26:221-226.

108 Vogelzang NJ, Porta C, Mutti L: New agents in the management of advanced mesothelioma. Semin Oncol 2005;32:336-350.

-109 Arber DA, Tamayo R, Weiss LM: Paraffin section detection of the c-kit gene product (CD117) in human tissues: value in the diagnosis of mast cell disorders. Hum Pathol 1998;29:498-504.

-110 Pietras K, Rubin K, Sjöblom T, et al: Inhibition of PDGF receptor signaling in tumor stroma enhances antitumor effect of chemotherapy. Cancer Res 2002;62:54765484.

-111 Ali Y, Lin Y, Gharibo MM, et al: Phase I and pharmacokinetic study of imatinib mesylate (Gleevec) and gemcitabine in patients with refractory solid tumors. Clin Cancer Res 2007;13:5876-5882.

112 Tsao AS, He D, Saigal B, et al: Inhibition of c-Src expression and activation in malignant pleural mesothelioma tissues leads to apoptosis, cell cycle arrest, and decreased migration and invasion. Mol Cancer Ther 2007;6:1962-1972.

113 Dudek A, Pang H, Kratzke A: A phase II study of dasatinib (D) in patients (pts) with previously treated malignant mesothelioma. J Natl Cancer Inst 2010;28:15.

- 114 Ramos-Nino ME, Testa JR, Altomare DA, et al: Cellular and molecular parameters of mesothelioma. J Cell Biochem 2006;98: 723-734.

115 Raymond E, Alexandre J, Faivre S, et al: Safety and pharmacokinetics of escalated doses of weekly intravenous infusion of CCI-779, a novel mTOR inhibitor, in patients with cancer. J Clin Oncol 2004;22: 2336-2347.

-116 Hartman ML, Esposito JM, Yeap BY, et al: Combined treatment with cisplatin and sirolimus to enhance cell death in human mesothelioma. J Thorac Cardiovasc Surg 2010;139:1233-1240.

117 Chang K, Pai LH, Pass H, et al: Monoclonal antibody K1 reacts with epithelial mesothelioma but not with lung adenocarcinoma. Am J Surg Pathol 1992;16:259-268.

118 Chang K, Pastan I: Molecular cloning of mesothelin, a differentiation antigen present on mesothelium, mesotheliomas, and ovarian cancers. Proc Natl Acad Sci USA 1996;93:136-140.

119 Hassan R, Bera T, Pastan I: Mesothelin: a new target for immunotherapy. Clin Cancer Res 2004;10:3937-3942.
120 Hassan R, Schweizer C, Lu KF, et al: Inhibition of mesothelin-CA-125 interaction in patients with mesothelioma by the anti-mesothelin monoclonal antibody MORAb009: implications for cancer therapy. Lung Cancer 2010;68:455-459.

121 Greillier L, Baas P, Welch JJ, et al: Biomarkers for malignant pleural mesothelioma: current status. Mol Diagn Ther 2008;12: 375-390.

122 Hassan R, Broaddus VC, Wilson S, et al: Anti-mesothelin immunotoxin SS1P in combination with gemcitabine results in increased activity against mesothelin-expressing tumor xenografts. Clin Cancer Res 2007;13:7166-7171.

123 Mikulski SM, Costanzi JJ, Vogelzang NJ, et al: Phase II trial of a single weekly intravenous dose of ranpirnase in patients with unresectable malignant mesothelioma. J Clin Oncol 2002:20:274-281.

-124 Mittelman A, Puccio C, Gafney E, et al: A phase I pharmacokinetic study of recombinant human tumor necrosis factor administered by a 5 -day continuous infusion. Invest New Drugs 1992;10:183-190.

125 Lejeune FJ, Lienard D, Matter M, et al: Efficiency of recombinant human TNF in human cancer therapy. Cancer Immun 2006; 6:6.

126 Gregorc V, Zucali PA, Santoro A, et al: Phase II study of asparagine-glycine arginine-human tumor necrosis factor alpha, a selective vascular targeting agent, in previously treated patients with malignant pleural mesothelioma. J Clin Oncol 2010;28: 2604-2611.

127 Kelly WK, O'Connor OA, Krug LM, et al: Phase I study of an oral histone deacetylase inhibitor, suberoylanilide hydroxamic acid, in patients with advanced cancer. J Clin Oncol 2005;23:3923-3931.

128 Paik PK, Krug LM: Histone deacetylase inhibitors in malignant pleural mesothelioma: preclinical rationale and clinical trials. J Thorac Oncol 2010;5:275-279.

129 Ramalingam SS, Parise RA, Ramanathan $\mathrm{RK}$, et al: Phase I and pharmacokinetic study of vorinostat, a histone deacetylase inhibitor, in combination with carboplatin and paclitaxel for advanced solid malignancies. Clin Cancer Res 2007;13:36053610.

130 Ramalingam SS, Belani CP, Ruel C, et al: Phase II study of belinostat (PXD101), a histone deacetylase inhibitor, for second line therapy of advanced malignant pleural mesothelioma. J Thorac Oncol 2009;4:97-101.

131 Marks PA, Richon VM, Miller T, et al: Histone deacetylase inhibitors. Adv Cancer Res 2004;91:137-168.

132 Scherpereel A, Berghmans T, Lafitte JJ, et al: Valproate-doxorubicin: promising therapy for progressing mesothelioma. A phase II study. Eur Respir J 2011;37:129-135.
133 Shapiro GI, Tibes R, Gordon MS, et al: Phase I studies of CBP501, a G2 checkpoint abrogator, as monotherapy and in combination with cisplatin in patients with advanced solid tumors. Clin Cancer Res 2011; 17:3431-3442.

134 Mulatero CW, Penson RT, Papamichael D, et al: A phase II study of combined intravenous and subcutaneous interleukin-2 in malignant pleural mesothelioma. Lung Cancer 2001;31:67-72.

135 Nowak AK, Lake RA, Kindler HL, et al: New approaches for mesothelioma: biologics, vaccines, gene therapy, and other novel agents. Semin Oncol 2002;29:82-96.

136 Astoul P, Picat-Joossen D, Viallat JR, et al: Intrapleural administration of interleukin-2 for the treatment of patients with malignant pleural mesothelioma: a phase II study. Cancer 1998;83:2099-2104.

-137 Caminschi I, Venetsanakos E, Leong CC, et al: Interleukin-12 induces an effective antitumor response in malignant mesothelioma. Am J Respir Cell Mol Biol 1998;19:738746.

138 Fennell DA, Chacko A, Mutti L: BCL-2 family regulation by the $20 \mathrm{~S}$ proteasome inhibitor bortezomib. Oncogene 2008;27: 1189-1197.

139 Sartore-Bianchi A, Gasparri F, Galvani A, et al: Bortezomib inhibits nuclear factorkappa B-dependent survival and has potent in vivo activity in mesothelioma. Clin Cancer Res 2007;13:5942-5951.

140 Gordon GJ, Mani M, Maulik G, et al: Preclinical studies of the proteasome inhibitor bortezomib in malignant pleural mesothelioma. Cancer Chemother Pharmacol 2008; 61:549-558.

141 Trandafir L, Ruffié P, Borel C, et al: Higher doses of alpha-interferon do not increase the activity of the weekly cisplatin-interferon combination in advanced malignant mesothelioma. Eur J Cancer 1997;33:19001902.

142 Upham JW, Musk AW, van Hazel G, et al: Interferon alpha and doxorubicin in malignant mesothelioma: a phase II study. Aust NZ J Med 1993;23:683-687.

143 Parra HS, Tixi L, Latteri F, et al: Combined regimen of cisplatin, doxorubicin, and alpha- $2 b$ interferon in the treatment of advanced malignant pleural mesothelioma: a phase II multicenter trial of the Italian Group on Rare Tumors (GITR) and the Italian Lung Cancer Task Force (FONICAP) Cancer 2001;92:650-656.

144 Halme M, Knuuttila A, Vehmas T, et al: High-dose methotrexate in combination with interferons in the treatment of malignant pleural mesothelioma. Br J Cancer 1999;80:1781-1785. 
145 Bretti S, Berruti A, Dogliotti L, et al: Combined epirubicin and interleukin-2 regimen in the treatment of malignant mesothelioma: a multicenter phase II study of the Italian Group on Rare Tumors. Tumori 1998; 84:558-561.

146 Hegmans JP, Hemmes A, Aerts JG, et al: Immunotherapy of murine malignant mesothelioma using tumour lysate-pulsed dendritic cells. Am J Respir Crit Care Med 2005;171:1168-1177.

147 Hegmans JP, Hemmes A, Hammad H, et al: Mesothelioma environment comprises cytokines and T-regulatory cells that suppress immune responses. Eur Respir J 2006;27: 1086-1095.

148 Hegmans JP, Veltman JD, Lambers ME, de Vries IJ, Figdor CG, Hendriks RW, Hoogsteden HC, Lambrecht BN, Aerts JG: Consolidative dendritic cell-based immunotherapy elicits cytotoxicity against malignant mesothelioma. Am J Respir Crit Care Med 2010;181:1383-1390.

149 Haas AR, Sterman DH: Novel intrapleural therapies for malignant diseases. Respiration 2012;83:277-292.

150 Tilleman TR, Richards WG, Zellos L, et al: Extrapleural pneumonectomy followed by intracavitary intraoperative hyperthermic cisplatin with pharmacologic cytoprotection for treatment of malignant pleural mesothelioma: a phase II prospective study. J Thorac Cardiovasc Surg 2009;138:405-411.
151 Sterman DH, Treat J, Litzky LA, et al: Adenovirus-mediated herpes simplex virus thymidine kinase/ganciclovir gene therapy in patients with localized malignancy: results of a phase I clinical trial in malignant mesothelioma. Hum Gene Ther 1998;9: 1083-1092.

152 Molnar-Kimber KL, Sterman DH, Chang $\mathrm{M}$, et al: Impact of preexisting and induced humoral and cellular immune responses in an adenovirus-based gene therapy phase I clinical trial for localized mesothelioma. Hum Gene Ther 1998;9:2121-2133.

153 Sterman DH, Recio A, Vachani A, et al: Long-term follow-up of patients with malignant pleural mesothelioma receiving high-dose adenovirus herpes simplex thymidine kinase/ganciclovir suicide gene therapy. Clin Cancer Res 2005;11:74447453.

154 Sterman DH, Recio A, Carroll RG, et al: A phase I clinical trial of single-dose intrapleural IFN-beta gene transfer for malignant pleural mesothelioma and metastatic pleural effusions: high rate of antitumor immune responses. Clin Cancer Res 2007; 13:4456-4466.

155 Hassan R, Ho M: Mesothelin targeted cancer immunotherapy. Eur J Cancer 2008;44: 46-53.

156 Hassan R, Zhang J, Pastan I: Antibodybased treatment for mesothelioma: clinical trials and laboratory studies. Lung Cancer 2006;54:S13.

157 Hassan R, Bullock S, Premkumar A, et al: Phase I study of SS1P, a recombinant antimesothelin immunotoxin given as a bolus I.V. infusion to patients with mesothelinexpressing mesothelioma, ovarian, and pancreatic cancers. Clin Cancer Res 2007; 13:5144-5149.
158 Li Q, Verschraegen CF, Mendoza J, et al: Cytotoxic activity of the recombinant antimesothelin immunotoxin, SS1(dsFv)PE38, towards tumor cell lines established from ascites of patients with peritoneal mesotheliomas. Anticancer Res 2004;24:1327-1335.

159 Armstrong DK, Laheru D, Ma WW, et al: A phase I study of MORAb-009, a monoclonal antibody against mesothelin in pancreatic cancer, mesothelioma, and ovarian adenocarcinoma (abstract). J Clin Oncol 2007;25:615s.

160 Brockstedt DG, Giedlin MA, Leong ML, et al: Listeria-based cancer vaccines that segregate immunogenicity from toxicity. Proc Natl Acad Sci USA 2004;101:13832-13837.

161 Thomas AM, Santarsiero LM, Lutz ER, et al: Mesothelin-specific CD8(+) T cell responses provide evidence of in vivo crosspriming by antigen-presenting cells in vaccinated pancreatic cancer patients. J Exp Med 2004;200:297-306.

162 Hassan R, Ebel W, Routhier EL, et al: Preclinical evaluation of MORAb-009, a chimeric antibody targeting tumor-associated mesothelin. Cancer Immun 2007;7:20.

163 Jantz MA, Antony VA: Pathophysiology of the pleura. Respiration 2008;75:121-133.

164 Froudarakis ME: Pleural diseases in the molecular era - time for more answers: introduction. Respiration 2012;83:2-4. 